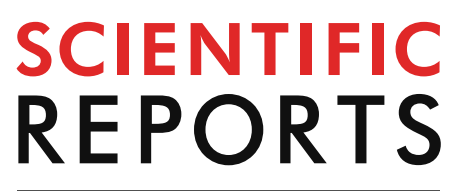

natureresearch

Check for updates

\title{
The effects of common
} variants in MDM2 and GNRH2 genes on the risk and survival of osteosarcoma in Han populations from Northwest China

\author{
Weilou Feng ${ }^{1}$, Zhi Wang ${ }^{2}$, Dongxu Feng ${ }^{1}$, Yangjun Zhu ${ }^{1}$, Kun Zhang ${ }^{1} \&$ Wei Huang ${ }^{1 \bowtie}$
}

Accumulating evidence has shown that both MDM2 and GNRH2 might be related to Osteosarcoma (OS) susceptibility. The study aimed to evaluate the effects of common variants in MDM2 and GNRH2 genes on the risk and survival of osteosarcoma in Han populations from Northwest China. In the study, we recruited 2292 subjects including 596 OS patients and 1696 healthy controls and genotyped 16 selected tag SNPs ( 6 from GNRH2 and 10 from MDM2). Genetic association analyses were performed at the genotypic and allelic levels. Survival curves were made for OS patients with different genotypes. Two SNPs, rs1690916 (MDM2, $P=0.0002)$ and rs3761243 (GNRH2, $P=0.0004)$, were identified to be significantly associated with OS risk. Moreover, SNP rs3761243 was strongly associated with pathological fracture $\left(P=2.61 \times 10^{-14}\right)$, metastasis $\left(P<2.2 \times 10^{-16}\right)$, and Enneking stage $\left(P<2.2 \times 10^{-16}\right)$ in the OS group. Furthermore, survival curves based on different genotypes of SNP rs3761243 were found to be significantly different $(P=0.0003)$, suggesting increased risk with more copies of $C$ alleles. Our results provide supportive evidence for genetic associations of MDM2 and GNRH2 genes with susceptibility to OS, and for the positive correlation of SNP rs3761243 in GNRH2 with the survival status of OS patients in Han populations from Northwest China.

Osteosarcoma (OS) is a common osteogenic tumor that occurs in children and adolescents under 18 years of age $^{1}$. Because of the high malignancy and aggressiveness of OS, the prognosis of OS is poor ${ }^{2}$. Surgery and chemotherapy are currently the main treatments for OS, but the 5-year survival rate of OS is still less than $50 \%{ }^{3}$. Hence, determining the pathogeny of OS and clarifying the molecular mechanism of OS are important problems to be solved. Previously obtained evidence has shown that the development of OS is complex and would be affected by many factors ${ }^{4,5}$. However, the fact that only a portion of individuals exposed to the same environment would develop OS has caught the attention of researchers, indicating that genetic factors are also risk factors for OS. Therefore, it is necessary to determine the susceptibility genes of OS through further research.

Previous research has shown that overexpression of MDM2 is found in a number of human cancers ${ }^{6}$. A case-control study has identified the significant association of genetic variants with OS in some candidate genes from biologically plausible pathways involved in growth, hormone metabolism, and DNA repair ${ }^{7}$. This study provided significant evidence for the associations of rs 1690916 in the MDM2 gene and rs3761243 in the GNRH2 gene with $\mathrm{OS}^{7}$. Additional evidence has also been reported suggesting that the single nucleotide polymorphism (SNP) rs 16909916 located in the $3^{\prime}$ untranslated region of $M D M 2$ is associated with susceptibility to OS in Europeans ${ }^{8,9}$. Nevertheless, some negative results for the association between rs16909916 and OS have also been reported. A case-control study did not observe any association between rs16909916 and OS in Spanish and Slovenian populations ${ }^{10}$. In addition, a meta-analysis including six populations also identified no association signals ${ }^{10}$. A previous study also detected a significant association of rs3761243 in GNRH2 with bone neoplasms ${ }^{11}$. Given that the molecular mechanisms of OS have not been determined, even though there is evidence of significant

${ }^{1}$ Department of Orthopaedic Trauma, HongHui Hospital, Xi'an Jiaotong University, 555 Youyi East Road, Beilin District, Xi'an 710053, Shaanxi, China. 'Department of Neonatology, Xi'an Children's Hospital, Xi'an, Shaanxi, China. ${ }^{\bowtie}$ email: weihuangxa@163.com 
associations with OS in Europeans, the contributions of MDM2 and GNRH2 to the pathogenesis of OS have not been fully explained to date. Thus, to characterize the roles of MDM2 and GNRH2 genes in OS predisposition, it is important to evaluate the relationship between them and OS risk in different ethnic populations.

To date, the roles of MDM2 and GNRH2 in the risk of OS in Han populations from Northwest China have not been explored. Thus, we aimed to examine the associations of common variants in MDM2 and GNRH2 with the OS susceptibility and to provide new clues for identifying individuals at high risk for OS and developing preventive strategies.

\section{Materials and methods}

Study subjects. We recruited a total of 2292 study subjects, consisting of 596 OS patients and 1696 controls from Honghui Hospital of Xi'an Jiaotong University and Xi'an Children's Hospital between January 2011 and April 2019. All OS patients were diagnosed based on standard histological and pathological evidence according to a consensus reached by at least two pathologists. The OS patients were followed after diagnosis, and death and month of survival of OS patients were recorded during the study period. Healthy controls included individuals who attend routine medical examination in the same hospitals mentioned above. To ensure the homogeneity of the genetic background, our study subjects were restricted to Xi'an natives with no immigration history within the last three generations. Informed consent forms were obtained from all participants. This study was performed in accordance with the ethical guidelines of the Helsinki Declaration of 1975 (revised in 2008) and was approved through the Ethics Committee of Honghui Hospital of Xi'an Jiaotong University (NO. 20190123).

SNP selection and genotyping. We selected tag SNPs for genotyping according to the following three criteria: (1) SNPs with minor allele frequency (MAF) $\geq 0.05$; (2) SNPs located within $\pm 3 \mathrm{~kb}$ gene regions of MDM2 and GNRH2; and (3) tag SNPs with $\mathrm{r}^{2} \geq 0.8$. In total, 16 tag SNPs were finally obtained for subsequent genotyping (6 from GNRH2 and 10 from MDM2). Basic information on these SNPs is summarized in Supplemental Table S1. We used commercial kits (Genomic DNA kit, Axygen Scientific, Inc., CA, USA) to extract genomic DNA from peripheral blood leukocytes. Then, a total of 16 SNPs were genotyped based on a highthroughput Sequenom MassARRAY platform. The genotype data of all SNPs in each subject were obtained. During the quality control phase, we randomly selected $5 \%$ of the samples for repeated genotyping ${ }^{12}$. The results are exactly the same as before, confirming the reliability of the quality of the genotyping data. During genotyping, the case/control status of each sample is unknown to all technicians ${ }^{13}$.

Statistical analysis. Hardy-Weinberg equilibrium (HWE) tests were conducted in the control group. Linkage equilibrium (LD) plots of the selected SNPs were constructed using Haploview ${ }^{14}$. Genetic association analyses were performed at the genotypic and allelic levels. For genotypic analysis, Cochran-Armitage trend tests were performed. For allelic analysis, $\chi^{2}$ tests were conducted. In addition, related analyses were preformed to examine the correlation between associated SNPs and several clinical features, including tumor location, pathological fracture, metastasis status and Enneking stage, within the OS patient group. Plink was utilized for genetic association analysis ${ }^{15}$. Bonferroni correction was used in multiple tests. For the scenario of SNP association analysis, when the $P$ value was less than $0.05 / 16=0.003125$, it was considered to be significant. Survival analyses were performed for OS patients with different genotypes of significant SNPs. Survival curves were made for OS patients with different genotypes. The Kaplan-Meier estimator and the log-rank test were utilized to evaluate the differences in survival curves for different genotypes. Cox models were fitted to estimate the hazard ratio of specific genotypes. The R project with the "survival" package (v3.2-3) was utilized for survival analysis ${ }^{16}$. In order to examine the functional consequence of the associated SNPs, we investigated the potential expression quantitative trait loci (eQTL) signals of the significant SNPs using data obtained from the GTEx database ${ }^{17}$. The data of eQTL signals for relevant SNPs on MDM2 and GNRH2 from multiple human tissues were downloaded and analyzed. In addition, we examined the significant SNPs in the PolymiRTS Database to explore the role of the SNPs in miRNA binding ${ }^{18}$.

\section{Results}

Demographic and characteristic information of the study subjects. In the present study, we recruited 596 OS patients and 1696 control subjects (Table 1). No significant differences could be identified for gender $(P=1.00)$ and age $(P=0.4374)$ between cases and controls. A significant difference was identified for family history between cases and controls $\left(P=6.80 \times 10^{-11}\right)$. Among the 596 OS patients, $114(19 \%)$ had tumors at their axial bones, and $482(81 \%)$ had tumors in their long bones. A total of 106 OS patients (18\%) had pathological fractures, and 490 patients (82\%) did not. Seventy-eight patients (13\%) had metastasis, and 518 patients (87\%) did not. Twelve percent of the OS patients (72) were in stage I of the Enneking staging system, and $75 \%$ (446 OS patients) and 13\% (78 OS patients) were in stage II and stage III, respectively.

Genetic association signals between genetic polymorphisms and OS risk. HWE tests were performed in control group. These SNPs were all in HWE in controls (Supplemental Table S1). LD plots are shown in Supplemental Figures S2 and S2. Among the 16 genotyped SNPs, two SNPs, rs1690916 (MDM2) and rs3761243 (GNRH2), were determined to be strongly associated with the risk of OS (Table 2). Similar significant results were also obtained in genotypic analyses. For SNP rs1690916, its A allele was correlated with the decreased risk of OS $\left(\mathrm{OR}=0.73[0.61-0.86], \chi^{2}=13.99, P=0.0002\right)$. On the other hand, the $\mathrm{C}$ allele of SNP rs3761243 was positively associated to an increased risk of $\mathrm{OS}\left(\mathrm{OR}=1.27[1.11-1.45], \chi^{2}=12.54, P=0.0004\right)$. Clear dose-dependent effects could be observed in genotypic analyses for both SNPs. The effect size increased when more copies of targeted alleles were present (Table 2). 


\begin{tabular}{|c|c|c|c|c|}
\hline Variables & Cases $(\mathrm{N}=596)$ & Controls $(\mathrm{N}=1696)$ & Statistics & $P$-values \\
\hline Age, mean + sd & $21.3 \pm 5.2$ & $21.5 \pm 5.3$ & $T=-0.78$ & 0.44 \\
\hline \multicolumn{5}{|l|}{ Gender, N (\%) } \\
\hline Male & $314(53)$ & $892(53)$ & & \\
\hline Female & $282(47)$ & $804(47)$ & $\chi^{2}=0.00$ & 1.00 \\
\hline \multicolumn{5}{|c|}{ Family history, N (\%) } \\
\hline Yes & $66(11)$ & $64(4)$ & & \\
\hline No & $530(89)$ & $1632(96)$ & $\chi^{2}=42.58$ & $6.80 \times 10^{-11}$ \\
\hline \multicolumn{5}{|c|}{ Tumor location, N (\%) } \\
\hline Axial & $114(19)$ & & & \\
\hline Long & $482(81)$ & & & \\
\hline \multicolumn{5}{|c|}{\begin{tabular}{|l|} 
Pathological fracture, N (\%) \\
\end{tabular}} \\
\hline Yes & $106(18)$ & & & \\
\hline No & $490(82)$ & & & \\
\hline \multicolumn{5}{|c|}{\begin{tabular}{|l|} 
Metastasis, N (\%) \\
\end{tabular}} \\
\hline Yes & $78(13)$ & & & \\
\hline No & $518(87)$ & & & \\
\hline \multicolumn{5}{|c|}{ Enneking stages, N (\%) } \\
\hline I & $72(12)$ & & & \\
\hline II & $446(75)$ & & & \\
\hline III & $78(13)$ & & & \\
\hline
\end{tabular}

Table 1. Demographic and characteristic information of the study subjects.

\begin{tabular}{|c|c|c|c|c|c|c|c|c|c|c|c|c|c|c|}
\hline & \multirow[b]{2}{*}{ CHR } & \multirow[b]{2}{*}{ Gene } & \multicolumn{6}{|c|}{ Genotypic Analyses } & \multicolumn{6}{|c|}{ Allelic Analyses } \\
\hline & & & Genotypes & Cases (\%) & \begin{tabular}{|l|}
$\begin{array}{l}\text { Controls } \\
(\%)\end{array}$ \\
\end{tabular} & OR [95\%] & $T$ & $P$ & Alleles & Cases (\%) & \begin{tabular}{|l|}
$\begin{array}{l}\text { Controls } \\
(\%)\end{array}$ \\
\end{tabular} & OR [95\%] & $\chi^{2}$ & $P$ \\
\hline \multirow{3}{*}{ rs1690916 } & \multirow{3}{*}{12} & \multirow{3}{*}{$M D M 2$} & AA & $13(2)$ & $93(5)$ & $\begin{array}{l}0.36 \\
{[0.20-0.64]}\end{array}$ & & & & & & & & \\
\hline & & & AG & $185(31)$ & $590(35)$ & $\begin{array}{l}0.80 \\
{[0.65-0.98]}\end{array}$ & & & A & $211(18)$ & $776(23)$ & \begin{tabular}{|l|}
0.73 \\
{$[0.61-0.86]$}
\end{tabular} & & \\
\hline & & & GG & $398(67)$ & $1013(60)$ & Ref & 14.00 & 0.0002 & G & $981(82)$ & $2616(77)$ & ref & 13.99 & 0.0002 \\
\hline \multirow{3}{*}{ rs3761243 } & \multirow{3}{*}{20} & \multirow{3}{*}{ GNRH2 } & CC & $168(28)$ & $377(22)$ & $\begin{array}{l}1.58 \\
{[1.22-2.06]}\end{array}$ & & & & & & & & \\
\hline & & & AC & $286(48)$ & $814(48)$ & $\begin{array}{l}1.25 \\
{[0.99-1.57]}\end{array}$ & & & C & $622(52)$ & $1568(46)$ & \begin{tabular}{|l}
1.27 \\
{$[1.11-1.45]$}
\end{tabular} & & \\
\hline & & & AA & $142(24)$ & $505(30)$ & Ref & 12.07 & 0.0005 & A & $570(48)$ & $1824(54)$ & ref & 12.54 & 0.0004 \\
\hline
\end{tabular}

Table 2. Significant SNPs identified in single marker based association analyses. CHR: chromosome.

Association between genetic polymorphisms and clinical features in OS patients. For the two significant SNPs, we examined their associations with several clinical features within the OS patient group (Table 3). SNP rs1690916 was not significantly associated with any clinical features in the OS group. However, SNP rs3761243 was significantly associated with pathological fracture $\left(\chi^{2}=62.56, P=2.61 \times 10^{-14}\right)$, metastasis $\left(P<2.2 \times 10^{-16}\right)$, and Enneking stage $\left(P<2.2 \times 10^{-16}\right)$ in the OS group. These results suggested clues for further survival analysis.

Survival analysis. Survival curves are shown in Fig. 1. Survival curves based on different genotypes of SNP rs3761243 were determined to be significantly different $(P=0.0003$, Fig. 1B). A similar difference was identified for survival curves made solely based on stage II OS patients (Fig. 1C). The Cox model for SNP rs3761243 was fitted for stage II OS patients with age, gender, pathological fracture, and metastasis status adjusted (Table 4). With the CC genotype serving as a reference, the hazard ratios with $95 \%$ confidence intervals for AC and AA genotypes were 0.89 [0.65-1.22] and 0.53 [0.36-0.78], respectively. Similar to the genotypic association analysis, a dose-dependent effect was also observed in the survival analysis. The hazard ratio increased when more copies of $\mathrm{C}$ alleles were present.

Functional consequences of the significant SNPs. Based on the results obtained from the PolymiRTS Database, the A allele of SNP rs1690916 could create a new binding site for miR-1303 on gene MDM2. This miRNA is encoded by gene MIR1303 located on 5q33.2. We explored the eQTL signals for both SNP rs1690916 


\begin{tabular}{|c|c|c|c|c|c|c|c|c|c|c|}
\hline & \multicolumn{3}{|c|}{ rs1690916 } & \multirow[b]{2}{*}{$\chi^{2 \mathrm{a}}$} & \multirow[b]{2}{*}{$P$} & \multicolumn{3}{|c|}{ rs3761243 } & \multirow[b]{2}{*}{$\chi^{2}$} & \multirow[b]{2}{*}{$P$} \\
\hline & AA & AG & GG & & & $\mathrm{CC}$ & AC & AA & & \\
\hline \multicolumn{11}{|c|}{ Tumor location, $\mathbf{N}(\%)$} \\
\hline Axial & 1 & 40 & 73 & & & 39 & 55 & 20 & & \\
\hline Long & 12 & 145 & 325 & - & 0.40 & 129 & 231 & 122 & 4.15 & 0.13 \\
\hline \multicolumn{11}{|c|}{ Pathological fracture, N (\%) } \\
\hline Yes & 2 & 32 & 72 & & & 63 & 31 & 12 & & \\
\hline No & 11 & 153 & 326 & - & 0.97 & 105 & 255 & 130 & 62.56 & $2.61 \times 10^{-14}$ \\
\hline \multicolumn{11}{|c|}{ Metastasis, N (\%) } \\
\hline Yes & 2 & 20 & 56 & & & 76 & 1 & 1 & & \\
\hline No & 11 & 165 & 342 & - & 0.57 & 92 & 285 & 141 & - & $<2.2 \times 10^{-16}$ \\
\hline \multicolumn{11}{|c|}{ Enneking stages, N (\%) } \\
\hline I & 4 & 28 & 40 & & & 2 & 2 & 68 & & \\
\hline II & 7 & 137 & 302 & & & 90 & 283 & 73 & & \\
\hline III & 2 & 20 & 56 & - & 0.06 & 76 & 1 & 1 & - & $<2.2 \times 10^{-16}$ \\
\hline
\end{tabular}

Table 3. Association between significant SNPs and clinical variables in cases. ${ }^{a}$ Fisher exact tests were performed for sparse data.

and rs3761243 on MDM2 and GNRH2, respectively (Fig. 2). Significant eQTL signals were identified for both SNPs in specific human tissues. SNP rs1690916 was strongly correlated with the gene expression of MDM2 in the spinal cord, whole blood and adipose tissue. SNP rs3761243 was determined to be significantly associated with the expression level of GNRH2 gene in the human testis.

\section{Discussion}

With the widespread application of sequencing technology, numerous susceptible genes were identified to be contributed to the risk of complex diseases, such as schizophrenia ${ }^{19-21}$. The gene MDM2, located at $12 \mathrm{q} 14.3-15$, is an important negative regulator of TP53, inhibiting the transcriptional activity of TP53 and enhancing proteolytic P53 degradation ${ }^{22,23}$. The importance of the p53 pathway in maintaining normal cell growth is supported by the fact that it is directly inactivated by mutations in the TP53 gene in approximately $50 \%$ of cancers ${ }^{24}$. Hence, MDM2 plays a key role in the development of malignant tumors. The GNRH2 (gonadotropin-releasing hormone 2) gene located at 20p13 participates in promoting gonadotropin synthesis and steroid hormone production ${ }^{25}$, and GNRH2 is also emerging as an important player in the biology of cancers, such as prostate cancer and ovarian cancer ${ }^{26,27}$.

Our study is the first one to explore the relationship between genetic polymorphisms of MDM2 and GNRH2 and susceptibility of OS based on populations with Chinese ancestry. In the present study, we obtained significant evidence to connect the two loci and OS. The direction of the genetic effect identified in our study was the same, and the effect size was also highly similar, compared to the study conducted by Mirabello et al. ${ }^{7}$. In this sense, our study could be considered a successful replication of this previous report in a different population. Moreover, it is difficult to draw solid conclusions only from SNP results ${ }^{28-30}$. Thus, considering the genetic heterogeneity of OS and differences in LD structure among different populations, this replication was meaningful. In addition, in our study, we moved one step further to show the dose-dependent pattern of the genotypic effects for OS susceptibility.

In bioinformatics analysis, we have identified that the A allele of SNP rs1690916 could create a new binding site for miR-1303 on gene MDM2. A previous study has showed that miR-1303 targets claudin-18 gene to modulate proliferation and invasion of gastric cancer cells ${ }^{31}$. In addition, according to our eQTL analysis, both significant SNPs rs1690916 and rs3761243 were significantly associated (at least in some specific types of human tissues) with gene expression of MDM2 and GNRH2, respectively. This finding indicated that both SNPs might have important functional consequences on the downstream translation from DNA variants to biological functions. Nevertheless, caution is required in interpreting the eQTL signals. In our study, our eQTL analyses were conducted solely based on data from a public database. As far as we know, there might be no OS patients included and tested in GTEx. However, the gene expression pattern and eQTL features might be significantly different in OS patients compared to control patients. In this sense, our eQTL signals identified based on GTEx data may only provide us with some clues but may not be used as solid evidence. In the future, an eQTL study based on OS patients is still needed to comprehensively examine the potential effect of SNPs rs 1690916 and rs3761243 on the gene expression of MDM2 and GNRH2.

We identified a strong correlation between the genotypes of SNP rs3761243 and the Enneking stages of OS in our samples. This observation is highly unusual because it seems that most OS patients in the stage I group had the AA genotype, while most patients in the stage III group had the CC genotype. Since the recruitment process of OS patients in our study was independent of the genotypes of SNP rs3761243 and the Enneking stages of the patients, a potential explanation for this observation is that SNP rs3761243 was highly correlated with Enneking stages in OS patients. However, more studies are still needed to replicate our results in other populations.

To date, no previous studies have focused on the survival of OS patients with different genotypes of SNP rs1690916 and rs3761243. Our survival analysis showed that the genotypes of SNP rs3761243 were significantly 


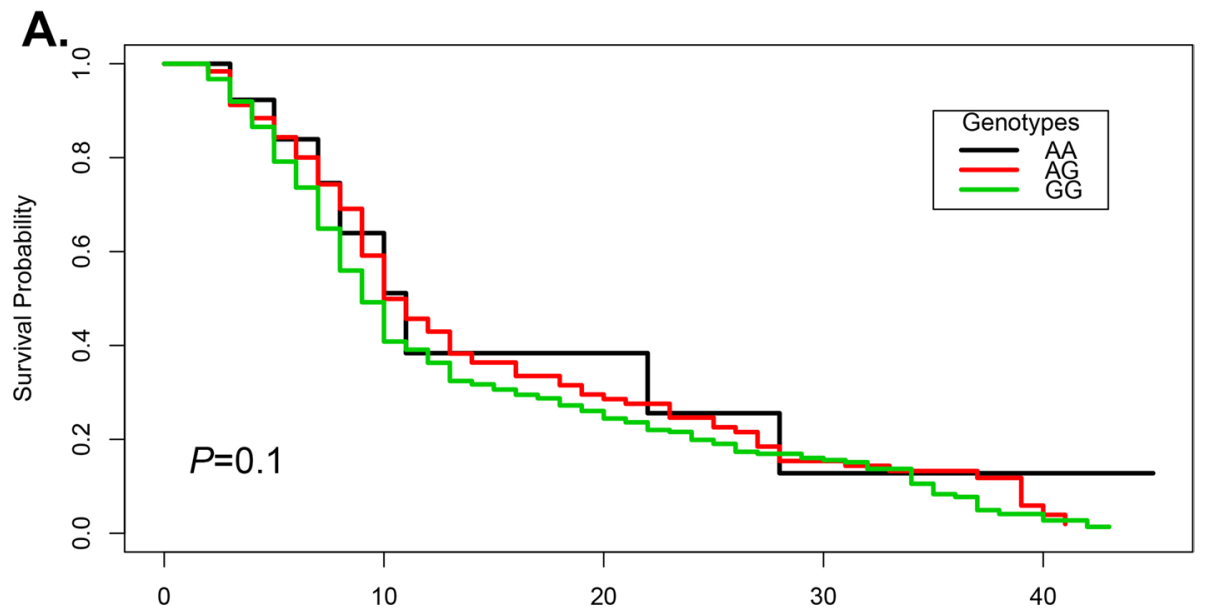

B.

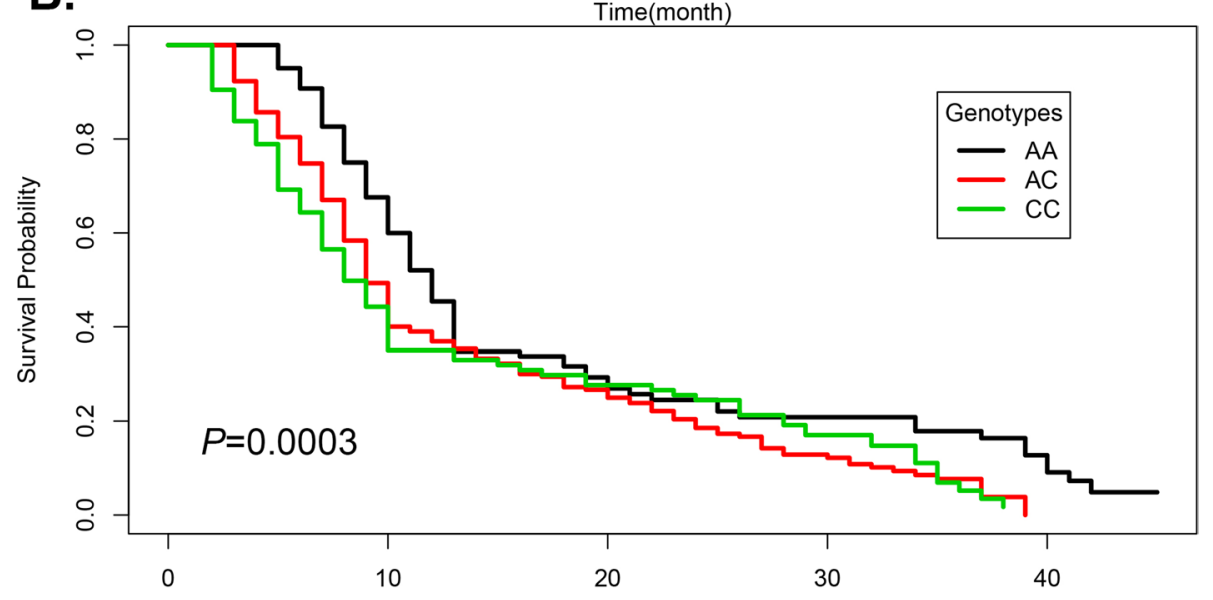

C. Time(month)

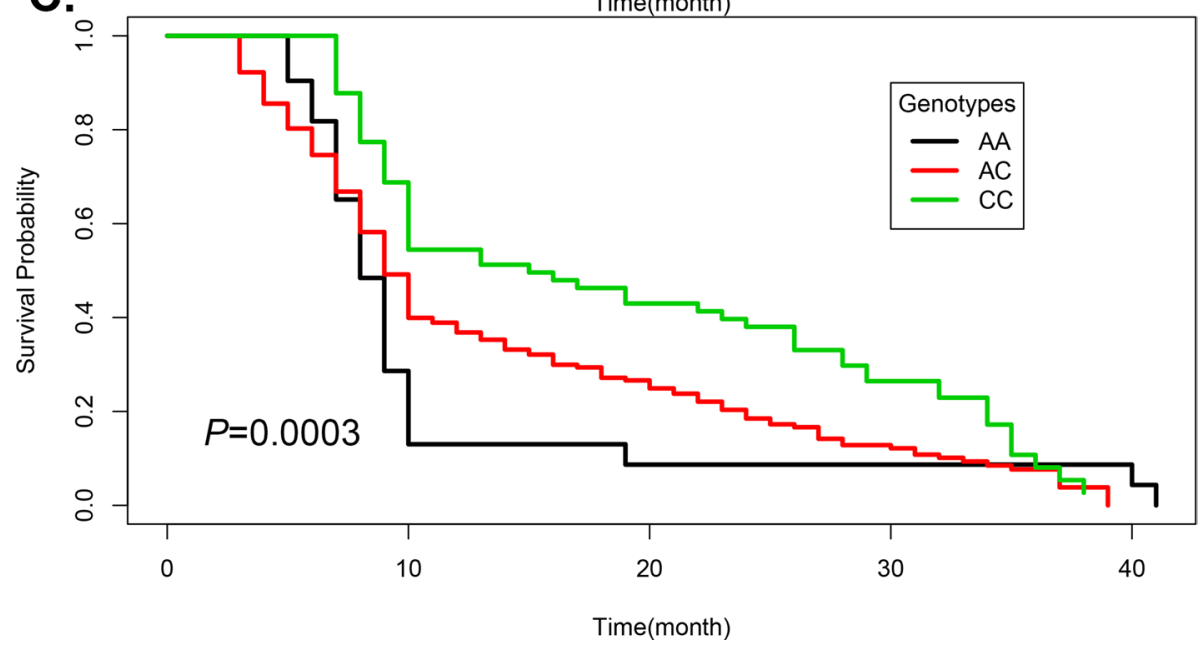

Figure 1. Survival curves for different groups of OS patients. (A) Survival curves categorized by genotypes of SNP rs1690916. (B) Survival curves categorized by genotypes of SNP rs3761243. (C) Survival curves categorized by genotypes of SNP rs3761243 in OS patients at Enneking stage II.

related to the survival of OS patients. In a further analysis focusing only on Enneking stage II OS patients, the AA genotype group had the poorest survival outcome. The HR estimated from the Cox model indicated that the C allele of SNP rs3761243 was positively correlated with a decreased hazard of OS death. This observation is highly interesting because the $\mathrm{C}$ allele at the SNP was correlated with an elevated risk of OS in genetic association analysis $(\mathrm{OR}=1.27$, Table 2), and it was also associated with elevated Enneking stages in OS patients (Table 3). Since SNP rs3761243 was not polymorphic in our Enneking stage I and Enneking stage III patients, we could not 


\begin{tabular}{|c|c|c|c|c|c|c|}
\hline SNP & CHR & GENE & Genotypes & Hazard ratio [95\%] & $\mathbf{Z}$ & $P$ \\
\hline \multirow{3}{*}{ rs3761243 } & \multirow{3}{*}{20} & \multirow{3}{*}{ GNRH2 } & $\mathrm{CC}(\mathrm{N}=90)$ & Ref & - & - \\
\hline & & & AC $(\mathrm{N}=286)$ & $0.89[0.65,1.22]$ & -0.72 & 0.4714 \\
\hline & & & AA $(\mathrm{N}=70)$ & $0.53[0.36,0.78]$ & -3.23 & 0.0012 \\
\hline
\end{tabular}

Table 4. Results of Cox model fitted for SNP rs3761243 using stage II osteosarcoma cases. Fracture and metastasis status were adjusted in Cox model.
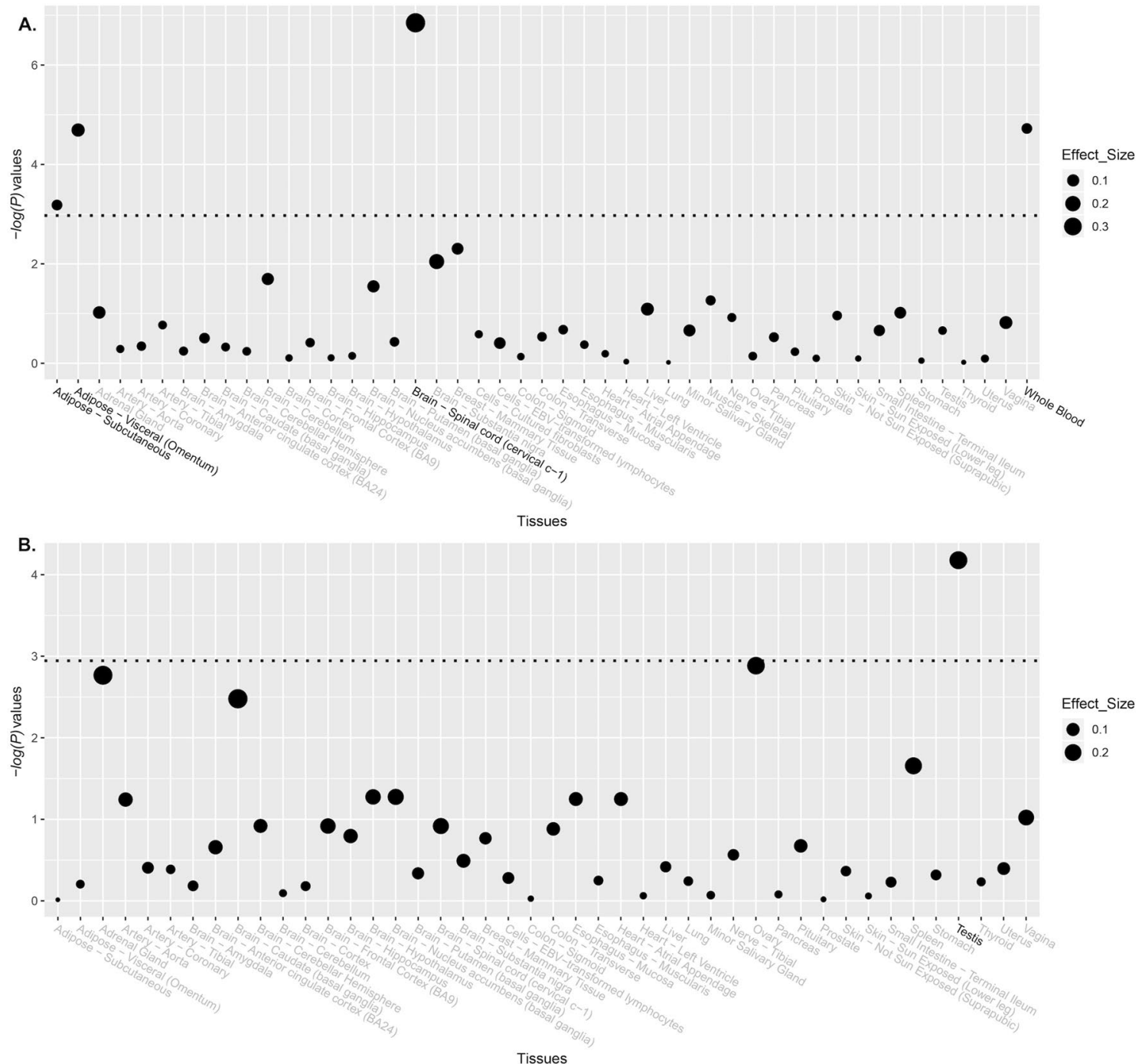

Figure 2. eQTL signals for both SNP rs1690916 and rs3761243 on MDM2 and GNRH2, respectively. (A) eQTL signals for both SNP rs1690916 on MDM2. (B). eQTL signals for both SNP rs3761243 on GNRH2.

comprehensively analyze the effect of genotypes on the survival of OS patients in the present study. The results obtained from stage II patients might be inaccurate and biased. In the future, a well-balanced study design is needed to analyze the genotypic effects on the survival of OS patients.

Our study has several limitations. First, we only selected and genotyped 16 SNPs for the two candidate loci, and the information coverage might not be enough to thoroughly examine the genetic susceptibility of the two loci contributing to the risk of OS. In addition, only common polymorphisms were included in this study. However, recent genetic studies suggest that low frequency and rare genetic variations may play a more critical role in the pathogenesis of complex diseases, especially for cancers ${ }^{32,33}$. Therefore, sequence-based studies would be desired to completely examine the genetic architecture of both MDM2 and GNRH2. Another potential limitation is that population stratification might be a confounded significant hit in the present study. Because of limited genotype data in the study, we hardly apply some standard statistical procedures that are commonly used in genome-wide association studies. Nevertheless, in the sample recruitment process, we restricted the genetic 
background of our study subjects by including only Xi'an natives with no immigration history in the last three generations. We think that this recruitment strategy at least partly addresses the potential confounding effect. In addition, the treatment protocols for patients with OS were not exactly the same in the study. These differences in treatment might affect the time of survival of these patients. Therefore, our results should be considered to be preliminary and validated in the future research.

The results of our study present significant evidence for a genetic association between OS susceptibility and MDM2 and GNRH2 genes in the sample of Han populations from Northwest China. More than genetic association with OS status, the genotypes of SNP rs3761243 in GNRH2 were also significantly associated with the survival status of OS patients at Enneking stage II. Our findings help to elucidate the screening and diagnosis of OS and the prognosis of OS after regular treatment.

Received: 31 March 2020; Accepted: 3 September 2020

Published online: 29 September 2020

\section{References}

1. Messerschmitt, P. J., Garcia, R. M., Abdul-Karim, F. W., Greenfield, E. M. \& Getty, P. J. Osteosarcoma. J. Am. Acad. Orthop. Surg. 17, 515-527. https://doi.org/10.5435/00124635-200908000-00005 (2009).

2. Geller, D. S. \& Gorlick, R. Osteosarcoma: a review of diagnosis, management, and treatment strategies. Clin. Adv. Hematol. Oncol. 8, 705-718 (2010).

3. Chou, A. J. \& Gorlick, R. Chemotherapy resistance in osteosarcoma: current challenges and future directions. Expert Rev. Anticancer Ther. 6, 1075-1085. https://doi.org/10.1586/14737140.6.7.1075 (2006).

4. Maximov, V. V. \& Aqeilan, R. I. Genetic factors conferring metastasis in osteosarcoma. Future Oncol. 12, 1623-1644. https://doi. org/10.2217/fon-2016-0014 (2016).

5. Yang, M. Prognostic role of pathologic fracture in osteosarcoma: evidence based on 1,677 subjects. J. Cancer Res. Ther. 11, $264-267$. https://doi.org/10.4103/0973-1482.151428 (2015).

6. Reifenberger, G., Liu, L., Ichimura, K., Schmidt, E. E. \& Collins, V. P. Amplification and overexpression of the Mdm2 gene in a subset of human-malignant gliomas without P53 mutations. Can. Res. 53, 2736-2739 (1993).

7. Mirabello, L. et al. A comprehensive candidate gene approach identifies genetic variation associated with osteosarcoma. $B M C$ Cancer 11, 209. https://doi.org/10.1186/1471-2407-11-209 (2011).

8. Toffoli, G. et al. Effect of TP53 Arg72Pro and MDM2 SNP309 polymorphisms on the risk of high-grade osteosarcoma development and survival. Clin. Cancer Res. 15, 3550-3556. https://doi.org/10.1158/1078-0432.Ccr-08-2249 (2009).

9. Naumov, V. A., Generozov, E. V., Solovyov, Y. N., Aliev, M. D. \& Kushlinsky, N. E. Association of FGFR3 and MDM2 gene nucleotide polymorphisms with bone tumors. B Exp. Biol. Med.153, 870-874. https://doi.org/10.1007/s10517-012-1847-9 (2012).

10. Bilbao-Aldaiturriaga, N. et al. A systematic review and meta-analysis of MDM2 polymorphisms in osteosarcoma susceptibility. Pediatr. Res. 80, 472-479. https://doi.org/10.1038/pr.2016.120 (2016).

11. Kushlinskii, N. E. et al. Associations of single nucleotide polymorphisms with malignant and borderline bone tumors. Klin Lab Diagn 58-60, 22-54 (2013).

12. Guan, F. et al. MIR137 gene and target gene CACNA1C of miR-137 contribute to schizophrenia susceptibility in Han Chinese. Schizophr Res. 152, 97-104 (2014).

13. Guan, F. et al. Association of PDE4B polymorphisms and schizophrenia in Northwestern Han Chinese. Hum. Genet. 131, 10471056 (2012).

14. Barrett, J. C., Fry, B., Maller, J. \& Daly, M. J. Haploview: analysis and visualization of LD and haplotype maps. Bioinformatics 21, 263-265. https://doi.org/10.1093/bioinformatics/bth457 (2005).

15. Chang, C. C. et al. Second-generation PLINK: rising to the challenge of larger and richer datasets. Gigascience 4, 7. https://doi. org/10.1186/s13742-015-0047-8 (2015).

16. R Development, C. T. R: a language and environment for statistical computing. 1, 12-21 (2007).

17. Consortium, G. T. The genotype-tissue expression (GTEx) project. Nat. Genet 45, 580-585. https://doi.org/10.1038/ng.2653 (2013).

18. Bhattacharya, A., Ziebarth, J. D. \& Cui, Y. PolymiRTS Database 3.0: linking polymorphisms in microRNAs and their target sites with human diseases and biological pathways. Nucleic Acids Res. 42, D86-D91. https://doi.org/10.1093/nar/gkt1028 (2014).

19. Zhang, T. X. et al. Voltage-gated calcium channel activity and complex related genes and schizophrenia: a systematic investigation based on Han Chinese population. J. Psychiatr. Res. 106, 99-105 (2018).

20. Han, W. et al. Relationship of common variants in CHRNA5 with early-onset schizophrenia and executive function. Schizophr Res. 206, 407-412 (2018).

21. Guan, F. et al. Evaluation of the relationships of the WBP1L gene with schizophrenia and the general psychopathology scale based on a case-control study. Am. J. Med. Genet. B Neuropsychiatr. Genet. 183, 164-171 (2020).

22. Shi, D. \& Gu, W. Dual roles of MDM2 in the regulation of p53: ubiquitination dependent and ubiquitination independent mechanisms of MDM2 repression of p53 activity. Genes Cancer 3, 240-248. https://doi.org/10.1177/1947601912455199 (2012).

23. Xirodimas, D. P., Saville, M. K., Bourdon, J. C., Hay, R. T. \& Lane, D. P. Mdm2-mediated NEDD8 conjugation of p53 inhibits its transcriptional activity. Cell 118, 83-97. https://doi.org/10.1016/j.cell.2004.06.016 (2004).

24. Muller, P. A. \& Vousden, K. H. p53 mutations in cancer. Nat Cell Biol 15, 2-8. https://doi.org/10.1038/ncb2641 (2013).

25. Parker, J. D., Malik, M. \& Catherino, W. H. Human myometrium and leiomyomas express gonadotropin-releasing hormone 2 and gonadotropin-releasing hormone 2 receptor. Fertil. Steril. 88, 39-46. https://doi.org/10.1016/j.fertnstert.2006.11.098 (2007).

26. Shiota, M. et al. The association of polymorphisms in the gene encoding gonadotropin-releasing hormone with serum testosterone level during androgen deprivation therapy and prognosis of metastatic prostate cancer. J. Urol. 199, 734-740. https://doi. org/10.1016/j.juro.2017.09.076 (2018).

27. Domanska, K. et al. Genetic profiles distinguish different types of hereditary ovarian cancer. Oncol. Rep. 24, 885-895. https://doi. org/10.3892/or.2010.885 (2010).

28. Sun, H. et al. Assessment of cognitive dysfunction in traumatic brain injury patients: a review. Forensic Sci. Res. 2, 174-179 (2017).

29. Zhang, Z. et al. Astrocytic clasmatodendrosis in the cerebral cortex of methamphetamine abusers. Forensic Sci. Res. 2, 139-144 (2017).

30. Guan, F. et al. Relationship of SNAP25 variants with schizophrenia and antipsychotic-induced weight change in large-scale schizophrenia patients. Schizophr. Res. 215, 250-255 (2020).

31. Zhang, SJ.et al. miR-1303 targets claudin-18 gene to modulate proliferation and invasion of gastric cancer cells. Dig. Dis. Sci. 59(8):1754-1763 (2014).

32. Verma, R. \& Sharma, P. C. Next generation sequencing-based emerging trends in molecular biology of gastric cancer. Am. J. Cancer Res. 8, 207-225 (2018). 
33. Balendran, S. et al. Next-generation sequencing-based genomic profiling of brain metastases of primary ovarian cancer identifies high number of BRCA-mutations. J. Neurooncol. 133, 469-476. https://doi.org/10.1007/s11060-017-2459-z (2017).

\section{Acknowledgements}

This research was supported by Shaanxi National Science Foundation (2018JM7048036). The funding sources had no role in the design of this study, the collection, analysis and interpretation of data, the writing of the report, or the decision to submit the paper for publication.

\section{Author contributions}

W.L.F. and W.H. conceived and designed the study. W.L.F. and Z.W. carried out candidate SNPs selection and statistical analyses. W.L.F., Z.F., D.X.F., Y.J.Z., K.Z. and W.H. conducted subject screening. Z.W., D.X.F., Y.J.Z. and K.Z. contributed to the collection and preparation of control DNA samples. W.L.F. wrote the paper.

\section{Competing interests}

The authors declare no competing interests.

\section{Additional information}

Supplementary information is available for this paper at https://doi.org/10.1038/s41598-020-72995-4.

Correspondence and requests for materials should be addressed to W.H.

Reprints and permissions information is available at www.nature.com/reprints.

Publisher's note Springer Nature remains neutral with regard to jurisdictional claims in published maps and institutional affiliations.

(c) (i) Open Access This article is licensed under a Creative Commons Attribution 4.0 International License, which permits use, sharing, adaptation, distribution and reproduction in any medium or format, as long as you give appropriate credit to the original author(s) and the source, provide a link to the Creative Commons licence, and indicate if changes were made. The images or other third party material in this article are included in the article's Creative Commons licence, unless indicated otherwise in a credit line to the material. If material is not included in the article's Creative Commons licence and your intended use is not permitted by statutory regulation or exceeds the permitted use, you will need to obtain permission directly from the copyright holder. To view a copy of this licence, visit http://creativecommons.org/licenses/by/4.0/.

(C) The Author(s) 2020 